
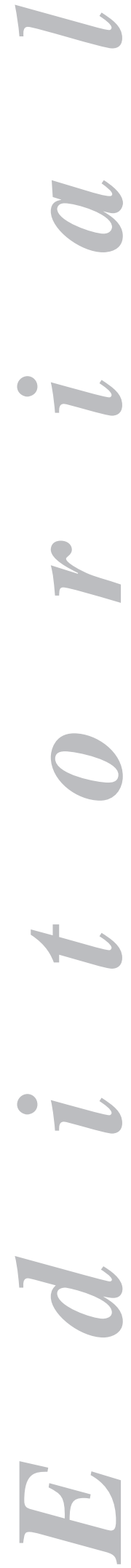

\section{The role of endoscopic ultrasonography in the biliary tract - A great friend of ERCP's}

Endoscopic ultrasonography (EUS) is unstoppably entering our Units' endoscopic armamentarium. The usefulness of this technique for patients with cancer or otherwise is increasing with an ongoing refinement of accuracy and a continuous expansion towards a varied therapeutic range. Being a mixed endoscopic and sonographic technique, EUS also benefits from advances such as Doppler, contrast enhancement, and elastography. Basically, EUS-related hardware may include radial and sectorial instruments as well as miniprobes; the latter are used through the working channels of various endoscopes, and may even enter the biliary tract (BT) in selected cases. Today, for instance, a miniprobe may be passed down a therapeutic enteroscope in complex situations to explore the BT following a Roux-en- $Y$ intestinal procedure. The ability to take the full potential of ultrasounds to the gut wall site nearest a lesion to be assessed provides the best approach possible, that is, an intraluminal approach using endoscopic instruments.

The BT may be explored with conventional abdominal ultrasounds, which represent the basic procedure and harmless initial exam for these patients. Already in this initial step whether dilating or not the intra- or extra-hepatic BT will guide a clinician's decisions, and that is why bedside sonography without delay, as suggested by Segura et al. (1), discriminates early management and in many centers guides patient admission. BT conditions involve a highly variable anatomical and morphological substrate, and their diagnosis and appropriate management are challenging: endoscopy, surgery or radiography combined with a comprehensive, mutidisciplinary approach to the underlying disease. In addition to such variation, also of interest for surgical treatment, the BT is a dynamic structure, and stones may be located at different sites or spontaneously expelled, a minor dilation may be seen when the sustaining parenchyma develops fibrosis (cirrhosis), a postural change may displace bile stones, etc. Time is highly relevant in the BT, and its correlation to clinical manifestations is vital.

Regarding diagnosis for BT diseases, another non-invasive technique to consider after abdominal US in the diagnostic algorithm is magnetic resonance cholangiography (MRC), which renders a rather reliable map of the BT, although with both technical (patient movement, metallic objects, sedation needed at times) and accuracy-related issues (small stones, short stenoses). Of course, endoscopic retrograde cholangio-pancreatography (ERCP) is superior to MRC in image quality given its dynamic contrast and action on the biliary tree (for example, with Fogarty balloon filling to better delimit a proximal area), thus better detecting extravasation, fistulas, etc.; however, ERCP has non-negligible complications and should be restricted for therapy. Hence, ERCP is -except for urgent cases such as serious cholangitis, etc.- a second-line technique following high diagnostic suspicion as a result of another non-invasive technique. EUS, however, is minimally invasive as a diagnostic technique with minimal com- 
plications similar to those of upper endoscopy, and may yield a lot of information, even more than MRC in BT conditions. In this issue of the Journal, Del Pozo et al. (2) assess EUS diagnostic accuracy in patients with a low or intermediate probability for BT disease, excluding those who had a dilated BT and/or a high suspicion for pathology. Abdominal US for these patients was normal, and based on well-established clinical criteria (3) a radial EUS was performed with ERCP (or surgery, e. g., for microlithiasis) indicated for positive findings. Over half of patients had BT disease, hence EUS was a rescue technique following abdominal US (and CT in some cases). In addition, EUS diagnosed other unnoticed processes outside the BT (chronic pancreatitis, peptic ulcer disease given the flexibility of modern, versatile equipment, etc.), and thus modified the natural history of BT disease in half of patients, and was deemed a safe technique. A subsequent ERCP treated confirmed findings.

In former comparative studies between MRC and EUS for BT disease, the superiority of EUS was clear for intermediate choledocholithiasis suspicion. The clinical probability of BT disease in a patient determines the subsequent diagnostic-therapeutic attitude, which could be stratified as follows: a) low probability: clinical follow-up, stop BT imaging; b) intermediate probability: indication of a non-invasive imaging technique (MRC and/or EUS) before the indication for ERCP is established; and c) high probability: assess ERCP directly.

MRC's early introduction and lower invasion versus EUS has favored its implementation; however, the increasing availability of EUS in our hospitals, and its higher sensitivity and specificity versus MRC for selected lesions (for instance, in the detection of small choledocholithiasis $(<5 \mathrm{~mm}$ ), leads to reconsider its use prior to ERCP in order to to support its indication should disease be found or discourage it and avoid a "blank" ERCP in the absence of findings. Even so, EUS is not exempt of limitations in the BT, for instance in the intrahepatic scenario (Klatskin's tumor evaluation).

Avoid unnecessary ERCPs with EUS, and indicate only those where BT disease data suggesting potential complications are really present represents improved management for these patients; it is about improving the healthcare circuit for these subjects, about an optimization of both techniques. In addition, EUS is an endoscopic technique and may thus be interventionist in the BT. This has a dual goal: either palliative endoscopic therapy (such as biliary drainage with EUS-guided prostheses) or a rendezvous technique to facilitate unsuccessful ERCP for BT cannulation, as it may introduce a guide to be later picked up by ERCP when completing therapy. There may also be interferences or artifacts that should be known regarding EUS. Some are spontaneous such as suprapapillary fistula following the passage of a stone, and other may be induced by ERCP itself when previously performed, as in the case of sphincterotomy, which may allow the passage of air in the BT, and limit US precision, or following a prosthesis placement, which may also limit the study and staging for cancer subjects, etc.

This is why timing and technique sequence are important. EUS is to be preferably used in a given cancer patient to be staged where therapeutic ERCP is planned; thus no technical interference will ensue, and ERCP will benefit from EUS findings, which may influence decision-making regarding the placement of a plastic or self-expandable prosthesis according to surgical irresectability and/or prognosis as established by EUS.

EUS and ERCP are condemned to get along with each other in the BT. All BT diseases where EUS discourages ERCP (unconfirmed stone suspicion, for example) are welcome for economy, shortened care process, and minimization of "unnecessary" complications. Currently we perform few "blank" ERCPs, most are to be performed with demanding therapy planning beforehand. 
However, learning ERCP and EUS is a high-level, complex, differentiated task. Acquiring competence in both is a training challenge for a new generation of young endoscopists. However, the current management of performance in endoscopy units or operating rooms demands effort to optimize these processes in an attempt to unify them into a single one, with just one sedation (with propofol by the endoscopist or with the help of an anesthesiologist), and only one decision that may (and should?) be made in one single procedure. Thus, for a patient with uncertain BT disease undergoing EUS, a decision whether ERCP is due or otherwise would be most efficient in its trail. If affirmative, given a potential failure of ERCP, EUS may in turn be used to rescue selected patients with failed BT cannulation under ERCP using the above-mentioned rendezvous. However, this involves complexity, as we should have sufficient infrastructure regarding materials, radiologic arch, ERCP tower, and EUS tower (in practice sometimes non-compatible videoprocessors), besides one or two practitioners competent in both techniques.

In the initial Spanish experience with EUS-guided ERCP, in a multicenter study in 16 Spanish hospitals by Vila et al. (4), a guide was successfully introduced using EUS in the BT in $90 \%$ of cases, with an overall clinical success rate of $93 \%$ using endoscopic rendezvous in 15 of 70 cases, with acceptable complications. ESCP (endosonography-guided cholangiopancreatography) success depended to a great extent on endoscopist training for ESCP and ERCP (more than 5 and 500 procedures performed, respectively). In this study ESCP by two endoscopists was preferable, which reflects the current-optimizable with training- situation in our setting.

For each "avoided" ERCP by a negative EUS procedure, the first endoscopist might think in the words of Martin Luther King, Jr: "At last, we will not remember our enemies' words, but we will remember our friends silence".

Maybe, if these new generation of excellent endoscopists who reach -as they shouldbeyond their masters acquires such complementary skills on EUS/ERCP, the primary benefit would be their becoming "friends of themselves", which is not too bad either.

Enrique Pérez-Cuadrado

Unit of Digestive Diseases. University of Murcia. Hospital Morales Meseguer. Murcia, Spain

\section{REFERENCES}

1. Segura JM. Ecografía digestiva. Ed. U. Autónoma de Madrid. 2011.

2. Del Pozo D, Tabernero S, Poves E, Sanz C, Beceiro I, Costero B, et al. Usefullness of endoscopic ultrasonography in the clinical suspicion of biliary disease. Rev Esp Enferm Dig 2011;103(7):345-8.

3. Canto MI, Chak A, Stellato T, Sivak MV. Endoscopic ultrasonography versus cholangiography for the diagnosis of choledocholithiasis. Gastrointest Endosc 1998; 47: 439-48.

4. Vila JJ, Vázquez-Sequeiros E, Perez Millán A et al. Experiencia inicial de la colangiopancreatografía guiada por ecoendoscopia (CPES): estudio multicéntrico nacional. Endoscopy 2010;42:997-1020. 